International Journal of Advanced Biological and Biomedical Research Available online at http:www.ijabbr.com

Volume 8, Issue 1 (2020) pp. 75-85

DOI: 10.33945/SAMI/IJABBR.2020.1.8

Original Article

\title{
Epidemiological and Genetic Overview of the Klebsiella pneumoniae Carbapenemases (KPCs) in K. pneumoniae Isolated from the Clinical Samples in Iran
}

\author{
Narjes Mohammadi Bandari ${ }^{1}$, Hossein Keyvani ${ }^{2}$, Mohsen Zargar ${ }^{1}$, Malihe \\ Talebi $^{3}$, Mohammad Reza Zolfaghari ${ }^{1}$ \\ ${ }^{1}$ Department of Biology, Qom Branch, Islamic Azad University, Qom, Iran \\ ${ }^{2}$ Department of Virology, Faculty of Medicine, Iran University of Medical Sciences, Tehran, \\ Iran \\ ${ }^{3}$ Department of Biology, Faculty of Medicine, Iran University of Medical Sciences, Tehran, \\ Iran \\ *Corresponding Author E-mail: Keyvanlab@yahoo.com
}

Received: 12 June 2019, Revised: 20 August 2019, Accepted: 30 August 2019

\begin{abstract}
Background: The prevalence of carbapenem-resistant Enterobacteriaceae, especially Klebsiella pneumoniae carbapenemase (KPC), has been recently reported worldwide. Therefore, there is an indispensable need for precise and rapid detection of these carbapenemases.
\end{abstract}

Objectives: This study was aimed to propose an accurate and rapid method for detecting K. pneumoniae carbapenemase genes from clinical samples, using reverse transcriptionpolymerase chain reaction (RT-PCR) and to evaluate the expression of these genes in the presence of $\beta$-lactam antibiotic by real-time PCR assay.

Methods: One hundred and eighty-one K. pneumoniae strains were collected from patients presenting to Firoozgar Hospital of Tehran, Iran. The strains were tested using the disk diffusion method, the modified Hodge test (MHT), and E-test minimum inhibitory concentration (MIC). Next, reverse transcription-PCR method was applied for the identification of bla oxA-23 and bla oxA-48 genes. Finally, expression of genes was measured by real-time PCR assay in the presence and absence of $\beta$-lactam antibiotic.

Results:Phenotypic testing showed a high level of antibiotic resistance, while the genotypic methods indicated the presence and expression of carbapenemase genes.

Conclusions: The findings suggest revisions in the current antibiotic therapy protocols, considering the high expression level of resistant carbapenemases to K. pneumoniae strains.

Key words: Bla oxA-23, Bla oxA-48, E-test, MHT, Real-time PCR.

\section{Introduction}

The World Health Organization (WHO) has recognized the antimicrobial resistance (AMR) as a major global health problem in recent decades (Prestinaci et al., 2015). AMR has also led to 
elevated mortality rates of nosocomial infections (Kritsotakis et al., 2017). The extensive dispersion of multidrug-resistant phenotypes of carbapenem-resistant Enterobacteriaceae compromises the control of healthcare-associated infections (Kelly et al., 2017). Genes causing AMR in bacteria are located on plasmids of various sizes. They can be transferred from one bacterium to another, from one person to another, and from one country to another (Zacharczuk et al., 2014). K. pneumonia is a Gram-negative bacillus, belongs to the Enterobacteriaceae family. It is an opportunistic pathogen, causing human nosocomial infections, septicemia, pneumonia, urinary tract infections, meningitis, diarrhea, and soft tissue infections (Mosavian et al., 2016). The rising multi-drug resistance (MDR) of K. pneumoniae isolates has led to limited treatment options for antibiotic therapy and infection control (García-Sureda et al., 2011). Changes in K. pneumoniae enzymes have made them resistant to antibiotics such as penicillins, cephalosporins, carbapenems, aminoglycosides, macrolides, and sulfamethoxazole (Doern et al., 2011). Among different metabolic processes, $\beta$-lactamase is recognized as the main survival and defense mechanism of these pathogenic bacteria against $\beta$-lactam antibiotics (Drawz and Bonomo, 2010). Carbapenemase-producing Enterobacteriaceae (CPE) in hospitalized patients has been a major concern for more than a decade (Tzouvelekis et al., 2014). Carbapenems comprise a class of betalactam antibiotics with significant antimicrobial activities (Nordmann et al., 2012). Carbapenemases can hydrolyze all ß-lactams, including imipenem, ertapenem, meropenem, and doripenem (Lee et al., 2016). Evidence suggests that misuse and overuse of carbapenems are correlated with the increasing antibiotic resistance of K. pneumoniae carbapenemase (KPC)producing bacteria (Haji Hashemi et al., 2016). Generally, carbapenemase enzymes, identified in Enterobacteriaceae, belong to one of three classes of $\beta$-lactamase, i.e., class A, B, or D. (Bush and Jacoby, 2010). $\beta$-lactamase class $A$ is becoming more prevalent in the Enterobacteriaceae family, with activities against carbapenems. Class D carbapenemases can be phylogenetically divided into different enzymes, such as bla oxA-23 and bla oxA-48 (Vasoo et al., 2013). These enzymes have been detected in K. pneumoniae strains, isolated from hospitalized patients. Clinical evidence has confirmed their potential to cause antibiotic resistance by hydrolyzing $\beta$-lactam antibiotics, including monobactams, carbapenems, and third-generation cephalosporins (Lee and Lee, 2010). Early and rapid detection of KPC-producing pathogens is essential for limiting the serious consequences of potential hospital infections by adopting proper infection control measures. This study proposes a rapid detection technique for identifying carbapenem resistance in hospitalized patients by examining the presence of carbapenemases in K. pneumoniae as a highly common bacterium. In this study, the emergence of Klebsiella species, with carbapenem-hydrolyzing class D $\beta$-lactamase genes (bla охA-23 and bla oxA-48), was reported in the clinical samples. Finally, the expression of genes in the presence and absence of $\beta$-lactam antibiotic was measured by realtime polymerase chain reaction (PCR) assay.

\section{Materials and methods}

\section{Study period and sample collection}

This cross-sectional study was conducted in Firoozgar Hospital, Tehran, Iran. Samples collected between March 2018 and December 2018. For this purpose, the patients were admitted to relevant wards. One hundred and eighty-one K. pneumoniae clinical samples were collected from the blood, stool, sputum, urine, burn wound, cerebrospinal fluid, trachea, skin lesion, eye discharge, abscess, and catheter. 


\section{Bacterial isolation}

The K. pneumoniae colonies were identified via Gram staining, as well as standard biochemical tests including indole, motility, citrate, urease, lactose fermentation, lysine decarboxylase, and MR-VP.

\section{Antimicrobial susceptibility testing: disk diffusion method}

Antibiotic discs were used to perform susceptibility tests via the disk diffusion method, comprised of ertapenem $(10 \mu \mathrm{g})$, imipenem $(10 \mu \mathrm{g})$, meropenem $(10 \mu \mathrm{g})$, doripenem $(10 \mu \mathrm{g})$, cefotaxime $(30 \mu \mathrm{g})$, ceftazidime $(30 \mu \mathrm{g})$, cefepime $(30 \mu \mathrm{g})$, cefoxitin $(30 \mu \mathrm{g})$, ceftriaxone $(30 \mu \mathrm{g})$, gentamicin $(10 \mu \mathrm{g})$, piperacillin $(100 \mu \mathrm{g})$, and aztreonam $(30 \mu \mathrm{g})$ based on the CLSI guidelines on the Mueller-Hinton agar plate. In the disk susceptibility test, K. pneumoniae ATCC 13883 strain was a positive control.

\section{Detection of the carbapenemase production: modified hodge test}

Carbapenem-resistant strains were exposed to the Modified Hodge Test (MHT) for the detection of carbapenemases production according to CLSI guidelines, 2016 (CLSI, 2016). After preparing a standard suspension of carbapenem-sensitive Escherichia coli (0.5 McFarland turbidity) in sterile saline, the solution was diluted in sterile saline (1:10). Then, using the standard disk diffusion method, it was inoculated on a Mueller-Hinton agar plate. The plate was left to dry for five minutes, and then, an ertapenem disk was placed at its center. Three to five colonies were picked from the organism using a swab and inoculated from the disk edge up to at least a 20-mm distance.

After incubating the plates overnight at $37{ }^{\circ} \mathrm{C}$, they were evaluated on the following day. They were then examined for improved growth around the organism streak at the intersection of the inhibition zone and the streak. The increased E. coli growth was indicative of carbapenemase formation by the tested organism, inactivating ertapenem. Also, an indentation was detected in the zone considering the presence of a cloverleaf-shaped or distorted inhibition zone. The CLSI guidelines interpret this phenomenon as positive in favor of carbapenemase production in the isolates.

\section{Minimum inhibitory concentration (MIC): epsilometery test}

The last test of resistance to carbapenems was an Epsilometery test intended for obtaining the minimum inhibitory concentration (MIC) in an imipenem disk. To this end, initially, a concentration of 0.5 McFarland standard was prepared from that MHT-positive isolates. Afterward, a sterile cotton swab was dipped into and pulled out slowly from the inoculums. The swab was rotated several times against the inner wall of the tube above the fluid level to removed excess liquid on Muller-Hinton agar plate. E-test strips (bioMérieux) were placed on the plate with "E"-end at the periphery of the plate. After incubating the plates for 24 hours at $37^{\circ} \mathrm{C}$, the MIC was read and recorded at the point where ellipse met the scale.

\section{Molecular identification of bla oxA-23 and bla oxA-48 genes in K. pneumoniae by reverse transcription-polymerase chain reaction (RT-PCR)}

The RT-PCR technique was applied to evaluate isolates with positive results on the modified Hodge test (MHT) for detection of bla oxA-23 and bla oxA-48 genes. RT-PCR was performed using specific primers for bla oxA-23 and bla oxA-48 to prevent false-positive results. Specific primer pairs, 
targeting bla oxA-23 and bla oxA-48 genes, were designed by IDT software and synthesized by Macrogen Company (Korea) (Table 1).

Table 1. Primer sequences of studied virulence genes in K. pneumoniae isolates and reaction setup for 2-step real-time PCR

\begin{tabular}{|c|c|c|c|c|}
\hline \multirow[t]{2}{*}{ Primer Sequences } & $\begin{array}{c}\text { RT- } \\
\text { PCR(step1) }\end{array}$ & \multicolumn{3}{|c|}{$\begin{array}{l}\text { Real-Time } \\
\text { PCR(step2) }\end{array}$} \\
\hline & Conditions & $\begin{array}{c}\text { Volume Re- } \\
\text { actions } \\
\end{array}$ & Conditions & $\begin{array}{c}\text { Volume Re- } \\
\text { actions } \\
\end{array}$ \\
\hline $\begin{array}{c}\text { bla oxA-23 } \\
\text { F- } \\
\text { GACACTAGGAGAAGCCATCAAG } \\
\text { R- } \\
\text { TGCATGAGATCAAGACCGATAC } \\
\text { T } \\
\text { bla oxA-48 } \\
\text { F- } \\
\text { ACATAAATCACAGGGCGTAGTT } \\
\text { R- CGAGGGCGATCAAGCTATT } \\
\text { rpo B } \\
\text { F-AACCCGCTGTCTGAGATTAC } \\
\text { R-GGCGTTTCGATCGGACATA } \\
\end{array}$ & $\begin{array}{c}1 \text { cycle } \\
25^{c} \ldots . . .10 \mathrm{~min} \\
47^{\circ} \text { c....60min } \\
70^{\circ} \text {.....10min }\end{array}$ & $\begin{array}{l}\text { Total RNA } 5 \mu \mathrm{l} \\
\text { Random } \\
\text { hexamer } 2 \mu \mathrm{l} \\
\mathrm{H}_{2} \mathrm{O} \text { up to } \\
10 \mu \mathrm{l}\end{array}$ & 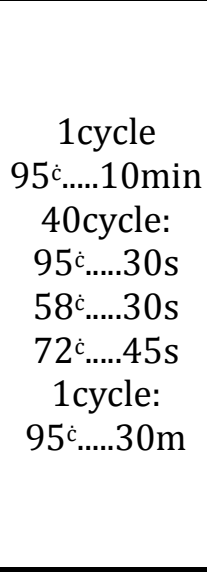 & $\begin{array}{c}\text { Real Q Plus } 2 \mathrm{x} \\
\text { Master Mix, } \\
\text { green: } 12.5 \mu \mathrm{l} \\
\text { Primer F+R } \\
\text { Primer: } 2 \mu \mathrm{l} \\
\text { Template } \\
\text { DNA: } 5 \mu \mathrm{l} \\
\text { Nuclease-free } \\
\text { water: } 5.5 \mu \mathrm{l} \\
\text { Total reaction } \\
\text { volume: } 25 \mu \mathrm{l}\end{array}$ \\
\hline
\end{tabular}

\section{Isolation of total RNA}

After inoculating the strains from glycerol stocks in nutrient agar $(2 \mathrm{~mL})$, they were grown at $37^{\circ} \mathrm{C}$ overnight. Then, the strains were sub-cultured in the nutrient medium $(5 \mathrm{~mL})$ and grown to the mid-exponential phase (OD600=1.5-2.0). Afterward, an aliquot of the culture $(0.25 \mathrm{~mL})$ was added to $2 \mathrm{~mL}$ of RNX-plus (Sinaclon, Iran). In addition, isolation of total RNA was performed based on the manufacturer's guidelines. DNase treatment was applied to remove the residual DNA with $20 \mathrm{U}$ of RQ1 DNase I. (Sinaclon, Iran).

\section{cDNA synthesis}

A reaction mixture, consisting of $5 \mu \mathrm{g}$ of RNA, was incubated in DEPC-treated water and $1 \mu \mathrm{L}$ of random hexamers (Pars Tous, Iran). Then, it was incubated for five minutes at $65{ }^{\circ} \mathrm{C}$ and cooled down on the ice. After $10 \mu \mathrm{L}$ of RT premixture (2X) was added, it was mixed by pipetting up and down gently in a total volume of $24 \mu \mathrm{L}$. Incubation was then performed at $25{ }^{\circ} \mathrm{C}$ for 10 minutes and at $47{ }^{\circ} \mathrm{C}$ for 60 minutes. Following that, the reaction was terminated by heating at $70{ }^{\circ} \mathrm{C}$ for 10 minutes and chilling on ice. The collected cDNA was stored at $-20{ }^{\circ} \mathrm{C}$ until further analysis.

\section{Real-time PCR assay}

Changes in bla gene expression were measured, using RT-PCR in the presence and absence of imipenem, in 36 antibiotic-resistant clinical strains containing two genes. Quantitative PCR (qPCR) was also performed according to the manufacturer's instructions. SYBR Green and 2X RT-PCR Master Mix Green were used in a No-Rox kit, and then, an RT-PCR detection system (Corbett, Australia) was used. Based on the melt curve analysis after 40 cycles, the presence of RT-PCR product was examined. The reference housekeeping gene was rpoB gene. The Rest software was used to calculate the gene expression, design of the graphs and the critical threshold cycle (CT). Then, the expression analysis was performed by relative measurement of 
mRNA expression in comparison with K.pneumoniae ATCC 13883 strain. That way, the comparative Ct $(\Delta \Delta \mathrm{Ct})$ method was applied to determine $\mathrm{Ct}$ values; fold differences were measured as 2- $-\Delta \Delta \mathrm{Ct}$.

\section{Statistical analysis}

To analyze the obtained data, SPSS version 23.0 (SPSS, Chicago, IL) using t-test was employed; for data analysis were performed of descriptive statistics (frequency, percentage, mean); the level of significance in the current study was $<0.05$.

\section{Results}

One hundred and eighty-one K. pneumoniae strains were collected from clinical samples such as aspirate $(n=65)$, sputum $(n=40)$, urine $(n=35)$, blood $(n=26)$ and other clinical samples $(\mathrm{n}=15)$. Cefepime and imipenem showed the highest and lowest rates of resistance, respectively. K. pneumoniae exhibited the highest susceptibility to meropenem $(38.12 \%)$ and the lowest susceptibility to cefepime (14.36\%). Therefore, all the samples were confirmed as multidrug-resistant (MDR) strains.

One-hundred strains, such as aspirate, sputum, urine, blood, and other clinical samples, were positive as KPC-producing in MHT (clover leaf-shaped) (Figure 1). The results of standard minimum inhibitory concentration (MIC) test were in agreement with those of MHT. The MICs ranged from $4 \mu \mathrm{g} / \mathrm{mL}$ to $6 \mu \mathrm{g} / \mathrm{mL}$ (MIC $\geq 4 \mu \mathrm{g} / \mathrm{mL}$ ). Therefore, these strains exhibited resistance to imipenem (Figure 2).

The findings showed that $51 \%(n=51)$ of strains were bla oxa -23 positive, and $84 \%(n=89)$ were bla oxA -48 positive; also, 92\% ( $\mathrm{n}=92)$ of the strains carried both genes (Table 2 and Figure 3).

Moreover, the gene expression of bla oxA-23 and bla oxA-48 was examined in this study. We observed the overexpression of these genes when induced by $2 \mathrm{mg}^{-1} \mathrm{~L}^{-1}$ of imipenem. The results demonstrated that imipenem caused 1.27 and 20.53-fold increase in the expression of genes, respectively.

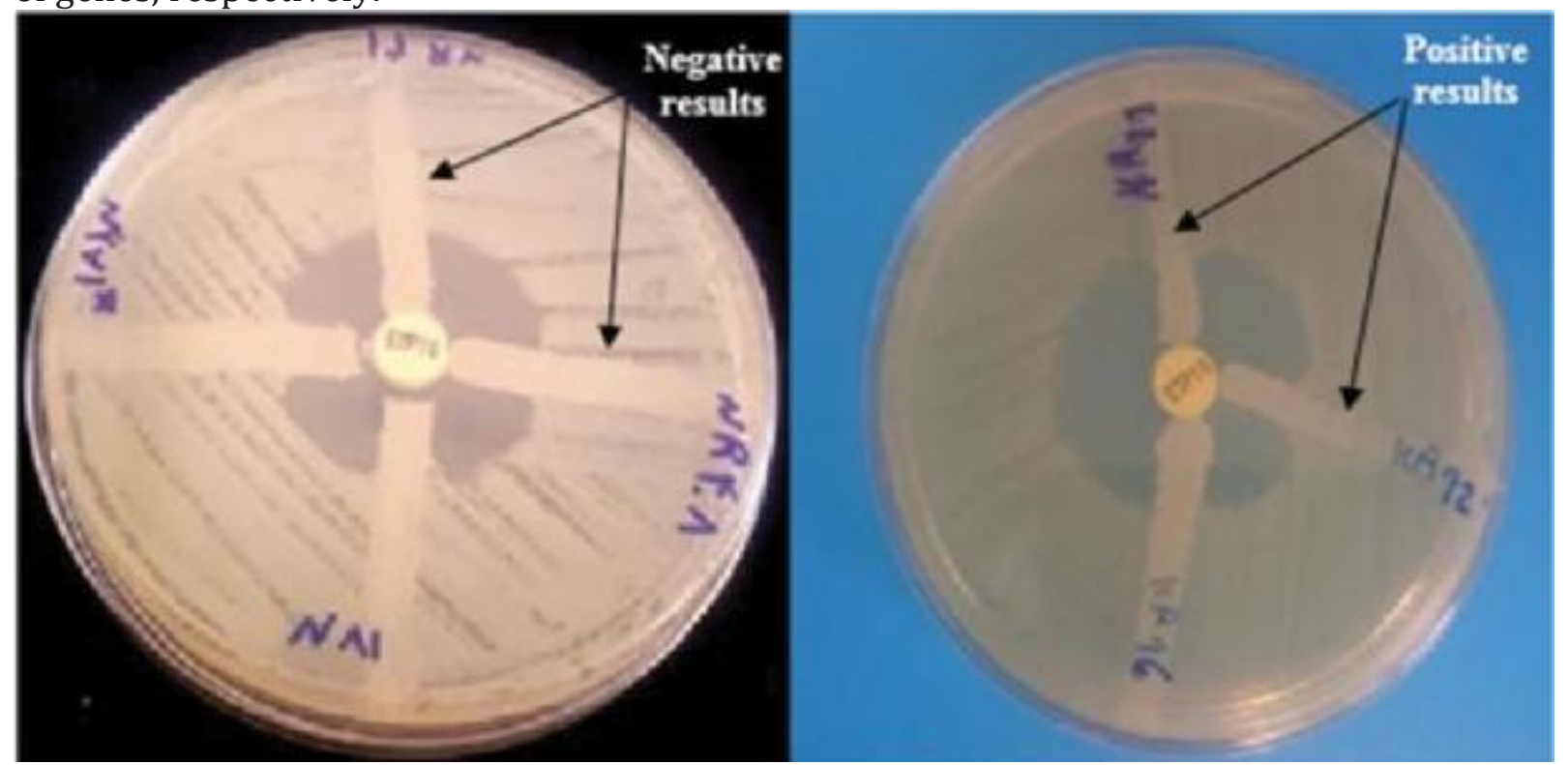

Figure 1. MHT test modified hodge test (MHT) showing positive and negative results 


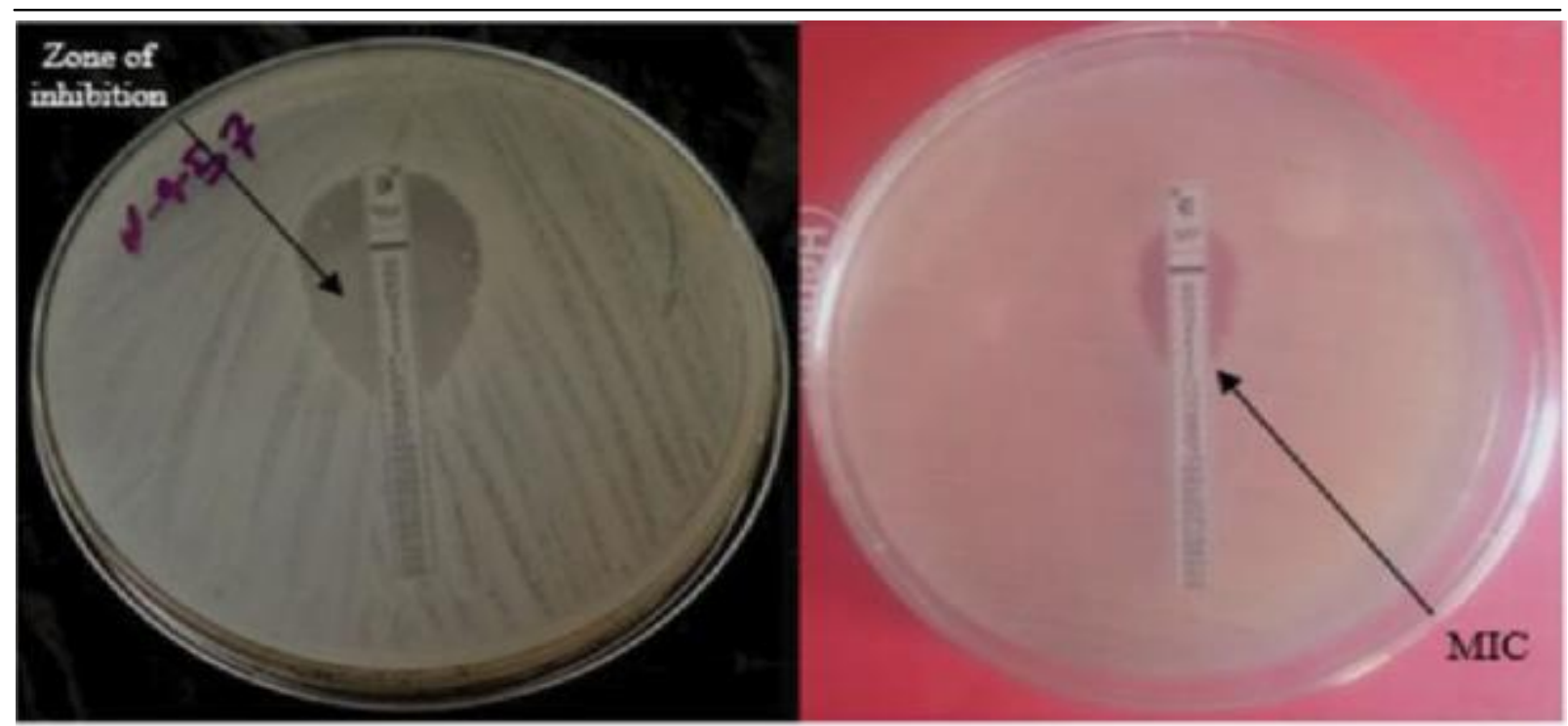

Figure 2. Epsilometery Test E-test inhibition ellipse showing the MIC at the intersection of the growth of the organism
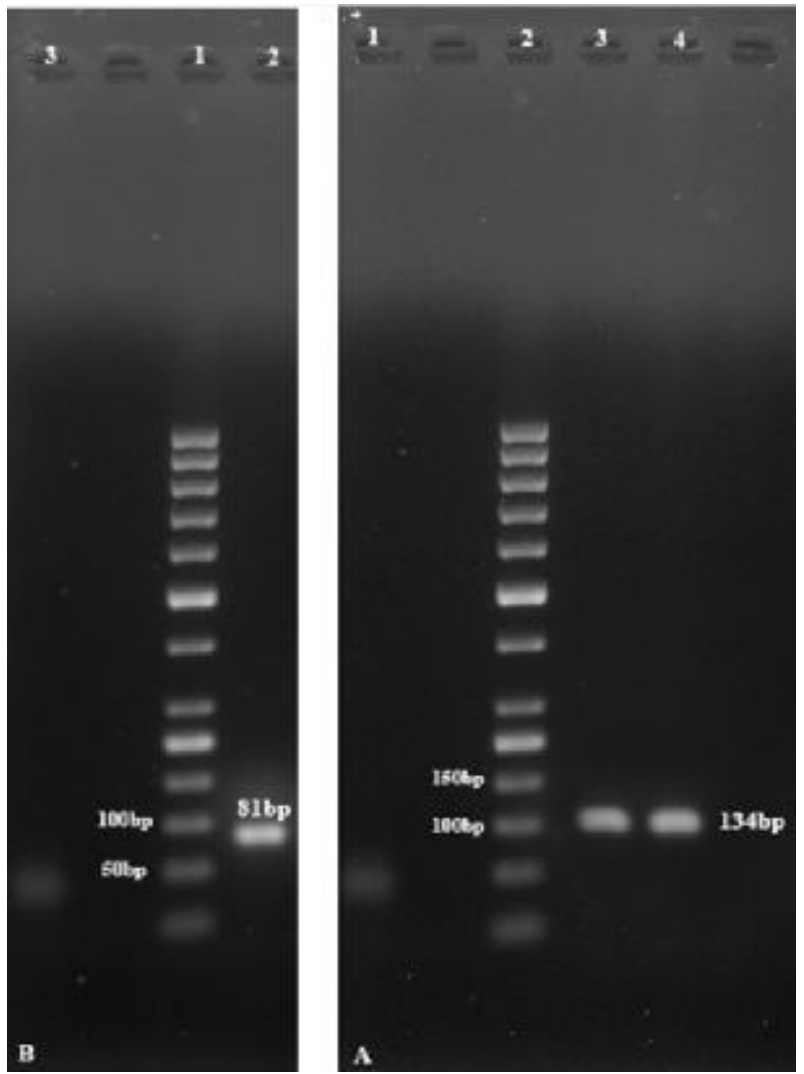

Figure 3. Agarose gel electrophoresis of PCR products

A. Gel electrophoresis shows the PCR product of bla oxA-48: Lane 1. Ctrl-, Lane 2. Gene ruler 25 bp DNA Ladder, Lane 3. Ctrl+ (K. pneumoniae ATCC 13883 strain), Lane 4. PCR product of bla oxA-48, $\mathbf{B}$. Gel electrophoresis shows the PCR product of bla oxA-23: Lane 1. Gene ruler 25 bp DNA Ladder, Lane 2. PCR product of bla oxa-23, Lane 3. Ctrl- 
Table 2. Antimicrobial resistance profile of K. pneumoniae isolates

\begin{tabular}{cccc}
\hline Sensitive(S) & Intermediate (I) & Resistant(R) & Antibiotic Name \\
\hline $66(36.5 \%)$ & $15(8.28 \%)$ & $100(55.2 \%)$ & Imipenem \\
$69(38.2 \%)$ & $10(5.24 \%)$ & $102(56.3 \%)$ & Meropenem \\
$65(36 \%)$ & $14(7.7 \%)$ & $102(56.3 \%)$ & Ertapenem \\
$66(37 \%)$ & $10(5.24 \%)$ & $105(58 \%)$ & Doripenem \\
$29(16.02 \%)$ & $2(1.1 \%)$ & $150(83.5 \%)$ & Cefotaxime \\
$31(17.12 \%)$ & $5(2.76 \%)$ & $145(80.1 \%)$ & Ceftazidime \\
$26(14.36 \%)$ & $3(1.65 \%)$ & $152(83.9 \%)$ & Cefepime \\
$46(25.41 \%)$ & $14(7.73 \%)$ & $121(66.8 \%)$ & Cefoxitin \\
$29(16.02 \%)$ & $4(2.2 \%)$ & $148(81.7 \%)$ & Ceftriaxone \\
$28(15.47 \%)$ & $3(1.65 \%)$ & $150(82.7 \%)$ & Gentamicin \\
$29(16.02 \%)$ & $4(2.2 \%)$ & $148(81.7 \%)$ & Piperacillin \\
$60(33.14 \%)$ & $13(7.18 \%)$ & $108(59.6 \%)$ & Aztreonam \\
\hline
\end{tabular}

\section{Discussion}

Nosocomial pathogens are important carbapenemase-producing K. pneumoniae strains. The prevalence of KPC-producing K. pneumoniae in hospitals is associated with increased mortality (Tzouvelekis et al., 2014). In addition to KPC-type carbapenemases, OXA-type enzymes are also found in K. pneumoniae. Currently, isolation and identification of bla oxa genes are a challenge for diagnostic laboratories. The presence of bla oxa merits special consideration, as the plasmid containing this gene leads to MDR and can be an epidemiological challenge, limiting the therapeutic options and increasing the mortality rate (Vasoo et al., 2013). Carbapenems are often applied as first-line treatment for drug-resistant Gram-negative organisms, while the increasing frequency of KPC organisms has decreased the efficacy of these antibiotics (Codjoe and Donkor, 2018). In this study, the lowest resistance was attributed to carbapenems based on the antibiotic susceptibility tests; this finding is consistent with the results of a study by Bina et al., which indicated the highest resistance to carbapenem (Bina et al., 2015). Considering the increasing spread of antimicrobial resistance (AMR) in hospital settings, the findings of the present study are similar to those reported by (Eilertson et al., 2017), and (Kontopoulou et al., 2010). These findings warrant more attention by the intensive care unit personnel to limit the potential risk of AMR in hospitals.

Our study showed that the resistant isolates to imipenem by E-test were 100 out of 181 (55.2\%), which is in agreement with the results of Al Hindi and El Shalakany (2016). On the other hand, in a study conducted by Gupta et al., in New York, USA, carbapenem resistance was reported by E-test in 10.8\% of the isolates (Gupta et al., 2011). In the present study, we found that the strains were positive for KPC-producing organisms, based on MHT, which is in agreement with the results of (Cury et al., 2012; Fattouh et al., 2015; Pasteran et al., 2010; Carvalhaes et al., 2009), as they found MHT (Cloverleaf test) to be a sensitive screening test for the detection of class A carbapenemases and discrimination of false-positive results for $\mathrm{K}$. pneumoniae.

The current study was performed for the first time in Iran using RT-PCR technique to determine the pattern of MDR and to investigate bla oxa- 23 and bla oxa-48 genes in resistant strains of K. pneumoniae according to phenotypic tests, as well as in vitro susceptibility tests. 
We showed that $84 \%$ of $\mathrm{K}$. pneumoniae isolates which were resistant to carbapenem were positive for bla oxa-23 gene and $92 \%$ isolates carrying bla oxA-48, which is in line with previous studies from Iraq Abbas and Jarallah confirmed the presence of bla oxa-23 gene based on the PCR assay (Abbas and Jarallah, 2017). This is the first outbreak of carbapenem-resistant K.pneumoniae isolates producing of bla OXA-23 is reported in Iran and the first report of K.pneumoniae isolates producing of bla oxA-48 in our hospital.

The first cases of OXA-48-producing K. pneumoniae strains in Iran reported by (Azimi et al., 2014). In Iran, there is another report of OXA-48 in K. pneumoniae by Solgi et al., according to their results, the bla oxA-48 gene was detected in 96 isolates (Solgi et al., 2018). Also, in another research in Iran, bla oxA-51, bla OXA-23, bla vIM, and bla KPC genes have been reported in A. baumannii (Azimi et al., 2015), that is, closest to our findings.

The antimicrobial resistance gene expression of $\mathrm{K}$. pneumoniae was measured by real-time PCR assay. We found that the expression rate of bla oxA-23 and bla oxA-48 in carbapenemresistant K.pneumoniae isolates from Firoozgar hospital is undoubtedly high.

Farivar et al., evaluated the expression level of oqxA and acrA genes among K. pneumoniae isolated. Real-time PCR assay showed a higher expression level of oqxAB ( 2.3 folds) and acrAB ( 4 folds) pumps in resistant strains, that is like with the result of our study (Farivar et al., 2016).

Also, the results of real-time PCR showed a two-fold increase in the expression of bla oxa-23 and bla oxa-48 genes in the presence of $2 \mathrm{mg}$. $\mathrm{L}^{-1}$ of imipenem. These results are consistent with a four-to eight-fold increase in MexX and mexY gene expression of Pseudomonas aeruginosa in the presence of tetracycline ( $2 \mathrm{mg} / \mathrm{L}$ ) (Dumas et al., 2006). Moreover, our results of the gene expression are in agreement with the study by Dhban et al, who showed the overexpression of 12 pilus genes in resistant Acinetobacter baumannii isolates by three folds when treated with a sub-MIC of imipenem (Dhabaan et al., 2016).

MHT was applied as a suitable method for evaluating the production of carbapenemase in the present study. Also, the RT-PCR method provided a convenient molecular tool for the detection and expression of bla oxa-23 and bla oxa-48 genes, providing a precaution for the actual outbreak. To the best of our knowledge, this study is the first report of the expression of bla oxa-23 and bla oxa-48 genes from K. pneumoniae strains in Iran. We also reported the emergence of an unprecedented oxa variant (oxa-23) in a K. pneumoniae isolate. Our findings revealed the high prevalence of oxa-23 and oxa-48 genes, encoding carbapenem resistance among $\mathrm{K}$. pneumoniae isolates in Firoozgar Hospital, Tehran, Iran.

\section{Conclusion}

Based on the present study and other similar researches, the emergence and spread of KPCproducing, extreme drug-resistant $\mathrm{K}$. pneumoniae isolates are increasing in hospital settings. Therefore, it is important to control the release of KPC-producing organisms by making changes in infection control strategies and administering appropriate antibiotic treatments in areas where patients have long hospital stays. Based on the findings, realtime PCR assay can reduce the turnaround time for infection control measures and detection of carbapenemase-producing organisms. Also, the current methods of laboratory diagnosis need to be improved to detect KPC resistance. Overall, the proposed method in this study can be considered an improvement to these methods. We hope that our findings can be applied for the implementation of an effective systematic strategy to manage 
infectious diseases and prevent the diffusion and dispersion of KPC-producing $\mathrm{K}$. pneumoniae in Iran.

\section{Acknowledgments}

The authors would like to thank the staff of the Microbiology Laboratory in Firoozgar Hospital Tehran and Keyvan research lab who contributed to this research

\section{References}

Abbas, FM, Jarallah, EM. (2017). Detection of OXA-23 among Carbapenem-Resistant Clinical Isolates of Klebsiella pneumoniae in Hilla. JUBPAS, 25(2):454-435.

Al Hindi, AA, El Shalakany, AH. (2016). Evaluation of a real-time PCR assay for the detection of the Klebsiella pneumoniae carbapenemase gene (blaKPC) in Enterobacteriaceae isolates from clinical samples in Menoufia University Hospitals. Egypt Afr. J. Microbiol., 10:13971407.

Azimi, L, Nordmann, P, Lari, AR, Bonnin, RA. (2014). First report of OXA-48-producing Klebsiella pneumoniae strains in Iran. GMS Hyg. Infect. Control, 9(1). doi: 10.3205/dgkh000227.

Azimi, L, Talebi, M, Pourshafie, MR, Owlia, P, Lari, AR. (2015). Characterization of carbapenemases in extensively drug resistance Acinetobacter baumannii in a burn care center in Iran. Int. J. Mol. Cell Med., 4(1):46.

Bina, M, Pournajaf, A, Mirkalantari, S, Talebi, M, Irajian, G. (2015). Detection of the Klebsiella pneumoniae carbapenemase (KPC) in K. pneumoniae Isolated from the Clinical Samples by the Phenotypic and Genotypic Methods. Iran J. Pathol., 10(3):199.

Bush, K, Jacoby, GA. (2010). Updated functional classification of $\beta$-lactamases. Antimicrob Agents Chemother., 54(3):969-976.

Carvalhaes, CG, Picão, RC, Nicoletti, AG, Xavier, DE, Gales, AC. (2009). Cloverleaf test (modified Hodge test) for detecting carbapenemase production in Klebsiella pneumoniae: be aware of false-positive results. J. Antimicrob. Chemother., 65(2):249-251.

Codjoe, F, Donkor, E. (2018). Carbapenem resistance: a review. Med. Sci., 6(1):1.

Cury, AP, Andreazzi, D, Maffucci, M, Caiaffa-Junior, HH, Rossi, F. (2012). The modified Hodge test is a useful tool for ruling out Klebsiella pneumoniae carbapenemase. Clinics, 67(12):1427-1431.

Dhabaan, GN, AbuBakar, S, Cerqueira, GM, Al-Haroni, M, Pang, SP, Hassan, H. (2016). Imipenem treatment induces expression of important genes and phenotypes in a resistant Acinetobacter baumannii isolate. Antimicrob Agents Chemother., 60(3):1370-1376.

Doern, CD, Dunne, WM, Burnham, CAD. (2011). Detection of Klebsiella pneumoniae carbapenemase (KPC) production in non-Klebsiella pneumoniae Enterobacteriaceae isolates by use of the Phoenix, Vitek 2, and disk diffusion methods. J. Antimicrob Chemother., 49(3), 1143-1147. 
Drawz, SM, Bonomo, RA. (2010). Three decades of $\beta$-lactamase inhibitors. Clin. Microbiol, 23(1):160-201.

Dumas, JL, Van Delden, C, Perron, K, Köhler, T. (2006). Analysis of antibiotic resistance gene expression in Pseudomonas aeruginosa by quantitative real-time-PCR. FEMS Microbiol. Lett., 254(2):217-225.

Eilertson, B, Chen, L, Chavda, KD, Kreiswirth, BN. (2017). Genomic characterization of two KPC-producing Klebsiella isolates collected in 1997 in New York City. Antimicrob Agents Chemother., 61(4):e02458-02416.

Fattouh, M, El-Din, AN, Omar, MA. (2015). Detection of Klebsiella pneumoniae carbapenemase (KPC) producing gram-negative superbugs: an emerging cause of multidrug-resistant infections in General Surgery Department of Sohag University Hospital, Egypt. Int. J. Curr Microbiol. App. Sci., 4(5):1-15.

García-Sureda, L, Juan, C, Doménech-Sánchez, A, Albertí, S. (2011). Role of Klebsiella pneumoniae LamB Porin in antimicrobial resistance. Antimicrob Agents Chemother., 55(4):1803-1805.

Guo, Y, Cen, Z, Zou, Y, Fang, X, Li, T, Wang, J, Chang, D, Su, L, Liu, Y, Chen, Y, Yang, R, Liu, C. (2012). Whole-genome sequence of Klebsiella pneumonia strain LCT-KP214. J. Bacteriol, 194:3281-3281.

Gupta, N, Limbago, BM, Patel, JB, Kallen, AJ. (2011). Carbapenem-resistant Enterobacteriaceae: epidemiology and prevention. Clin. Infect Dis., 53(1):60-67.

Haji Hashemi, B, Farzanehkhah, M, Dolatyar, A, Imani, M, Farzami, M, Rahbar, M, Hajia, M. (2012). A study on prevalence of KPC-producing from Klebsiella pneumoniae using Modified Hodge Test and CHROMagar in Iran. Ann. Biol. Res., 3(12):5659-5664.

Kelly, AM, Mathema, B, Larson, EL. (2017). Carbapenem-resistant Enterobacteriaceae in the community: a scoping review. Int.J. Antimicrob. Agents, 50(2):127-134.

Kontopoulou, K, Protonotariou, E, Vasilakos, K, Kriti, M, Koteli, A, Antoniadou, E, Sofianou, D. (2010). Hospital outbreak caused by Klebsiella pneumoniae producing KPC-2 $\beta$-lactamase resistant to colistin. J. Hosp. Infec., 76(1):70-73.

Farivar, AS, Nowroozi, J, Eslami, G, Sabokbar, A, Hashemi, A. (2016). The study of antibiotic resistance among Klebsiella pneumoniae and expression level of oqxA and acrA genes by using real-time PCR. Res. Med., 40(1):42-48.

Kritsotakis, EI, Flora Kontopidou, EA, Roumbelaki, M, Ioannidou, E, Gikas, A. (2017). Prevalence, incidence burden, and clinical impact of healthcare-associated infections and antimicrobial resistance: a national prevalent cohort study in acute care hospitals in Greece. Infect. Drug Resist., 10:317.

Lee, CR, Lee, JH, Park, KS, Kim, YB, Jeong, BC, Lee, SH. (2016). Global dissemination of carbapenemase-producing Klebsiella pneumoniae: epidemiology, genetic context, treatment options, and detection methods. Front. Microbiol., 7:895. 
Lee, JH, Lee, SH. (2010). Carbapenem resistance in gram-negative pathogens: Emerging non-metallo-carbapenemases. Res. J. Microbiol., 5(7):607-628.

Nordmann, P, Poirel, L, Dortet, L. (2012). Rapid detection of carbapenemase-producing Enterobacteriaceae. Emerg. Infect. Diseases, 18(9):1503.

Pasteran, F, Mendez, T, Rapoport, M, Guerriero, L, Corso, A. (2010). Controlling falsepositive results obtained with the Hodge and Masuda assays for detection of class A carbapenemase in species of Enterobacteriaceae by incorporating boronic acid. J. Clin. Microbiol., 48(4):1323-1332.

Prestinaci, F, Pezzotti, P, Pantosti, A. (2015). Antimicrobial resistance: a global multifaceted phenomenon. Pathog. Glob. Health, 109(7):309-318.

Solgi, H, Badmasti, F, Giske, CG, Aghamohammad, S, Shahcheraghi, F. (2018). Molecular epidemiology of NDM-1-and OXA-48-producing Klebsiella pneumoniae in an Iranian hospital: clonal dissemination of ST11 and ST893. J. Antimicrob Chemother., 73(6):15171524.

Tzouvelekis, L, Markogiannakis, A, Piperaki, E, Souli, M, Daikos, G. (2014). Treating infections caused by carbapenemase-producing Enterobacteriaceae. Clin. Microbiol. Infect., 20(9):862-872.

Vasoo, S, Cunningham, SA, Kohner, PC, Simner, PJ, Mandrekar, JN, Lolans, K, Patel, R. (2013). Comparison of a novel, rapid chromogenic biochemical assay, the Carba NP test, with the modified Hodge test for detection of carbapenemase-producing Gram-negative bacilli. J. Clin. Microbiol., 51(9):3097-3101.

Zacharczuk, K, Piekarska, K, Szych, J, Zawidzka, E, Sulikowska, A, Wardak, S, Jagielski, M, Gierczyński, R. (2011). Emergence of Klebsiella pneumoniae coproducing KPC-2 and 16S rRNA methylase ArmA in Poland. Antimicrob. Agents Chemother., 55(1):443-446.

How to cite this article: Narjes Mohammadi Bandari, Hossein Keyvani, Mohsen Zargar, Malihe Talebi, Mohammad Reza Zolfaghari, Epidemiological and Genetic Overview of the Klebsiella pneumoniae Carbapenemases (KPCs) in K. pneumoniae Isolated from the Clinical Samples in Iran. International Journal of Advanced Biological and Biomedical Research, 2020, 8(1), 75-85. Link: http://www.ijabbr.com/article 36267.html 